\title{
Musculoskeletal and cutaneous sarcoidosis: exuberant case report ${ }^{*}$
}

\author{
Natalie Haddad ${ }^{1}$ \\ Ana Maria França Corbett ${ }^{1}$
}

\author{
Jayme de Oliveira Filho ${ }^{1}$ \\ Ana Carolina Franco Tebet ${ }^{1}$
}

\author{
Kassila da Rosa Nasser ${ }^{1}$ \\ Mariana Lacerda Junqueira Reis ${ }^{1}$
}

\begin{abstract}
Sarcoidosis is a multisystem granulomatous disease of unknown cause. The osteoarticular involvement in sarcoidosis is rare and is often associated with cutaneous and long-standing chronic multisystem disease. More common in black women, osseous sarcoidosis is difficult to diagnose, with an incidence of 3 to $13 \%$. The most characteristic radiological clinical picture evidences rounded, well-defined cysts, with no periosteal reaction and without peripheral sclerosis. The small bones of hands and feet are the most frequently involved sites. This report aims to demonstrate a rare case of osteoarticular sarcoidosis with characteristic clinical presentation, and highlight the importance of detecting osteoarticular involvement in this pathology.
\end{abstract}

Keywords: Bone resorption; Granuloma; Granulomatous disease, chronic; Joint deformities, acquired; Lumpy skin disease; Sarcoidosis; Skin abnormalities; Skin diseases; Skin and connective tissue diseases

\section{INTRODUCTION}

Sarcoidosis is a complex multisystem granulomatous disease of unknown cause. The lung is the most affected organ, however virtually any organ can be involved, like the skin and, more rarely, the osteoarticular system. The prevalence is slightly higher in women. Age distribution follows a bimodal pattern with peaks in the $3^{\text {rd }}$ and $5^{\text {th }}$ decades. Black individuals tend to present a more severe disease, refractory and with extrapulmonary involvement. Its physiopathology is not yet understood. Likewise, the manner in which the extrapulmonary sarcoidosis lesions occur has not yet been clarified. The skin is involved in about 20 to $35 \%$ of the cases, a fact that places the dermatologist in an important role in diagnosing the disease. The most characteristic cutaneous manifestation of sarcoidosis is Lupus pernio, which affects mainly women and the central region of the face in the form of papular nodular lesions of purplish-red color, with telangiectasias. Osteoarticular manifestations of sarcoidosis are rare, predominating on fingers and toes and frequently accompanied by cutaneous lesions. The most characteristic radiological clinical picture evidences rounded, well-defined cysts, with no periosteal reaction and without peripheral sclerosis.

\section{CASE REPORT}

An 83-year-old female patient, black, hypertensive and diabetic, complained about nasal lesions and deformities on hands for 16 years. At the examination she presented purplish erythematous nodules with telangiectasias located on the nose, with "apple jelly" aspect to diascopy (Figure 1). On hands she presents important deformities, with shortened, swollen and bent fingers and presence of nodules in interphalangeal and metacarpophalangeal articulations, painful to palpation (Figure 2). She reported that this situation started around 16 years before, the nasal lesion being painless; a short time afterwards the hand joints became painful and swollen. Through the years she started presenting deformities on hands, which became progressively aggravated. She reported that at this time she was mistakenly diagnosed with Hansen's and received treatment for such disease, with no improvement. Radiography of hands showed destructive lytic bone lesions of the distal phalanges, with pathological fractures and presence of well-defined cystic lesions on proximal and distal phalanges (Figure 3). Thorax radiography did not present changes. Histopathology of nasal lesion showed granulomatous dermatitis of tuberculoid type (sarcoid granu-

\footnotetext{
Received on 13.08.2013.

Approved by the Advisory Board and accepted for publication on 23.08.2013.

Work performed at Universidade de Santo Amaro (UNISA) - Santo Amaro (SP), Brazil.

Conflict of interest: None

Financial funding: None

Universidade de Santo Amaro (UNISA) - Santo Amaro (SP), Brazil.
} 
loma) (Figure 4). We started the treatment with systemic corticoid, with fast improvement of articular pain and decrease of cutaneous lesion.

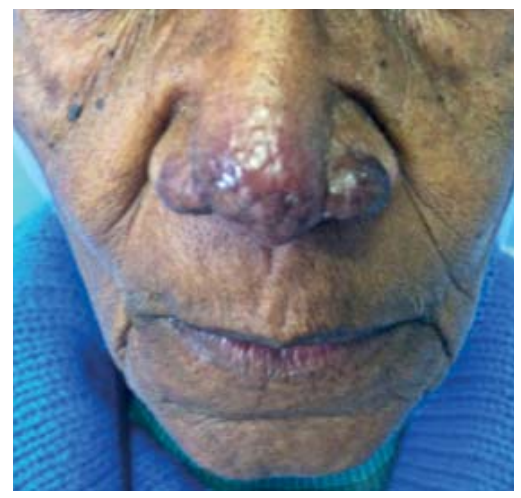

Figure 1:

Classical lesion of cutaneous sarcoidosis on nasal region: purplish erythematous nodule with telangiectasias

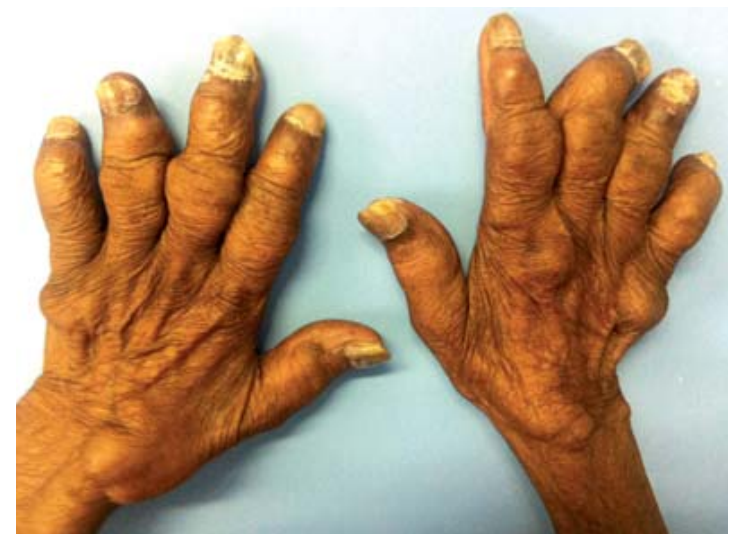

Figure 2: Exuberant deformities in hands: articular nodules, deviations and shortening of fingers

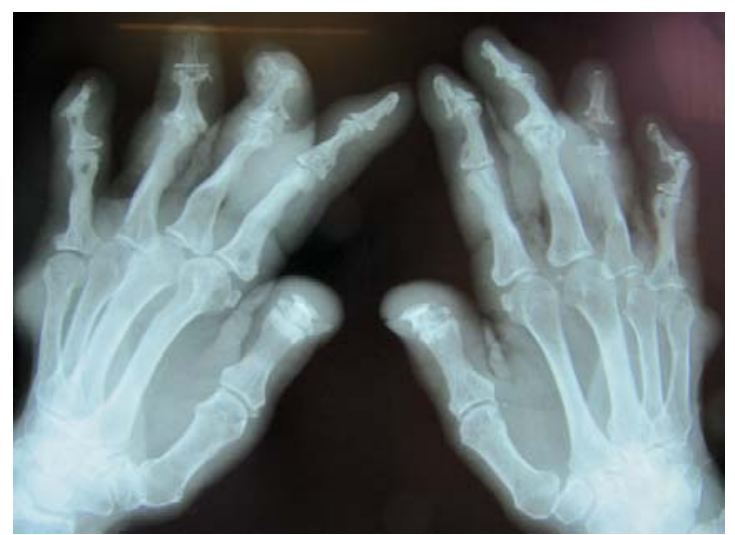

FIGURE 3: Radiography: lytic and cystic osseous lesions on phalanges

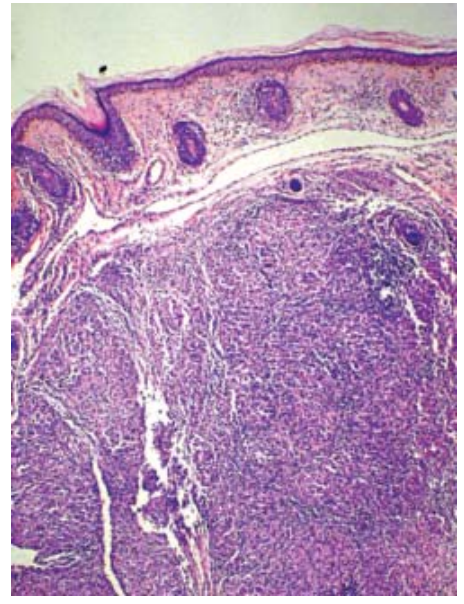

Figure 4:

Anatomopathologic examination of cutaneous lesion: Sarcoid granuloma

\section{DISCUSSION}

Osteoarticular involvement in sarcoidosis is rare and is associated with long-standing chronic multisystem disease. ${ }^{1}$ More frequent in black women, it is a pathology of difficult diagnosis, for it is usually asymptomatic. ${ }^{2}$ The incidence varies from 3 to $13 \%$ and it is clinically significant in 2 to $5 \%$ of the cases. ${ }^{1,2}$ The forms of osseous involvement vary from lytic and cystic lesions (the last two being the most common) to invasive lesions with trabecular irregularity, destructive lesions with periosteal reaction and disseminated lesions, which may cause diagnostic confusion with myeloma and bone metastases. Patients with osseous involvement frequently have pulmonary diseases (80$90 \%$, which was not found in our case, and are more prone to having cutaneous involvement, especially lupus pernio (48-70\%), as in the case presented. ${ }^{3}$ The small bones of hands and feet are the most frequently involved sites. ${ }^{3}$ The most common form of clinical presentation is dactylitis, which occurs mainly in black patients, and is very similar to the form seen in psoriatic arthritis. ${ }^{4}$ The so-called Jüngling disease or multiple cystoid osteitis corresponds to the most characteristic radiological picture which evidences round cysts, well-delimited, with no periosteal reaction and without peripheral sclerosis. ${ }^{5}$ Periosteal reaction is rare, and in severe cases, fragmentation and bone resorption may occur, besides presence of soft tissue edema. Cysts are small focal lesions, however, large cysts may involve the cortex as well as the bone marrow predisposing to pathological fracture. ${ }^{1}$

Acute arthritis occurs in $10 \%$ to $40 \%$ of patients with sarcoidosis, mainly in the initial phases of the disease, possibly being the first manifestation or as part of Lofgren's syndrome. ${ }^{6}$ This syndrome is characterized by triad hilar adenopathy, acute polyarthritis and erythema nodosum. ${ }^{2}$ It is more frequent in 
women and it is believed that it arises from the presence of circulating immune complexes. Most of the time it is symmetric and oligoarticular. The most involved articulations are ankles, however knees, wrists and elbows may also be involved. Other articulations, such as small articulations of hands and feet, hips and sternoclavicular and sacroiliac joints are less frequently involved. Normally, it is self-limited and recurrences are uncommon. ${ }^{1,7}$

Chronic sarcoid arthritis is uncommon, being usually associated with long-standing sarcoidosis in patients with cutaneous involvement. It presents in the polyarticular form, involving hands, wrists, shoulders, ankles and knees. It is frequently accompanied by lupus pernio, chronic uveitis and progressive pulmonary sarcoidosis; erythema nodosum is almost never found. ${ }^{8,9}$ It is characterized by remission and exacerbation periods or it can be persistent. Articular deformity is rare, occurring in $1 \%$ to $4 \%$ of the patients with sarcoidosis, and it has already been described as Jaccoud's arthropathy, which is characterized by metacarpal deviation of fingers, metacarpophalangeal subluxations and "swan neck" and boutonniere deformities, besides " $Z$ " thumb deformity. The different forms of chronic arthritis that may occur among patients with sarcoidosis are: non-deforming arthritis with granulomatous synovitis; Jaccoud-type deformity (non-erosive); articular edema adjacent to the osseous lesion by sarcoidosis; dactylitis (soft tissue edema in one or more digits).

The natural history of Osteoarticular Sarcoidosis varies from rare cases of recovery to progressive cases with self-amputation. ${ }^{1,6}$ It presents poor response to treatment. Due to its rarity, there are few studies guiding its therapy. Corticoids are still the basis of therapy, but other immunosupressors may be used. Biological drugs seem to be a promising future approach. The case presented demonstrates the clinical and radiological findings characteristic of Osteoarticular Sarcoidosis and its association with the cutaneous clinical picture. The detection of osteoarticular involvement in sarcoidosis may help the clinician in the diagnosis and the start of adequate therapy avoiding possible associated morbidities.

\section{REFERENCES}

1. Zisman DA, Shorr AF, Lynch JP 3rd. Sarcoidosis involving the musculoskeletal system. Semin Respir Crit Care Med. 2002;23:555-70.

2. Dalton PEC, Arruda LHF. Noninfectious granulomas: sarcoidosis. An Bras Dermatol. 2007;82:559-71.

3. Abril A, Cohen MD. Rheumatologic manifestations of sarcoidosis. Curr Opin Rheumatol. 2004;16:51-5.

4. Jacyk WK. Cutaneous sarcoidosis in black South Africans. Int J Dermatol. 1999;38:841-5.

5. Souza EM; Rosa SP. Afecções granulomatosas não infecciosas. In: Junior $\mathrm{WB}, \mathrm{Di}$ Chiachio N, Criado PR. Tratado de Dermatologia. São Paulo: Ed. Atheneu; 2010. p. 517-30.

6. Sharma SK, Soneja M, Sharma A, Sharma MC, Hari S. Rare manifestations of sarcoidosis in era modern of new diagnostic tools. Indian J Med Res. 2012;135:621-9. Mehrota P, Muller M, Higgins B, Zammit-Maemple I. An unusual cause of widespread lyic bone lesions caused by sarcoidosis. J Radiol Case Rep. 2011;5:1-7. Mckee PH, Calonje E, Granter SR. Pathology of the skin with clinical correlations. 3rd ed. Philadelphia: Elsevier Mosby; 2005.

9. Ahmed I, Harshad SR. Subcutaneous sarcoidosis: is it a specific subset of cutaneous sarcoidosis frequently associated with systemic disease? J Am Acad Dermatol. 2006;54:55-60.

\author{
MAILING ADDRESS: \\ Natalie Haddad \\ Rua Professor Enéas de Siqueira Neto, 340 \\ Jardim das Embuias \\ 04829-300 - São Paulo - SP \\ Brazil \\ E-mail: natahaddad@hotmail.com
}

How to cite this article: Haddad N, Oliveira Filho J, Nasser KR, Corbett AMF, Tebet ACF, Reis MLJ. Musculoskeletal and cutaneous sarcoidosis: exuberant case report. An Bras Dermatol. 2014;89(4):660-2. 